\title{
Perspectives on Workforce Development Needs for Community Health Workers (CHWs): Results from a Statewide Survey of CHW Employers
}

\author{
Melissa Dunn ${ }^{1}\left[\right.$ [ Emily Peterson Johnson ${ }^{1} \cdot$ Beatrice Smith ${ }^{1} \cdot$ Maria Cooper $^{1} \cdot$ Nimisha Bhakta $^{1}$
}

Accepted: 27 March 2021 / Published online: 13 April 2021

(C) The Author(s), under exclusive licence to Springer Science+Business Media, LLC, part of Springer Nature 2021

\begin{abstract}
We conducted a survey of community health worker (CHW) employers in Texas to understand the employment context and workforce development needs of Texas CHWs. An electronic, mixed-methods survey was emailed to $841 \mathrm{CHW}$ employers across Texas in Spring 2020. The survey consisted of 51 questions. The response rate was $22 \%(n=182)$. Responses were analyzed using SPSS, Microsoft Excel, and N.Vivo. We found that most CHW employers directly employ their CHWs, and CHWs are typically part of a multidisciplinary healthcare team. Most respondents required their CHWs be certified by the state's health department and have at least a high school diploma or GED. The most common services that CHWs provide are health education/promotion and information referral. The main health issues that CHWs address are diabetes, hypertension, and mental/behavioral health. Current CHW workforce development needs include continued training on topics including chronic disease self-management and health promotion. CHW employers differ in their capacity to implement workforce development activities. There is significant variety in the employment context and workforce development needs of CHWs across Texas. Results reinforce previous findings on the need for specialized, continuing training for CHWs and the development of pathways, resources, and opportunities that could advance the CHW profession even more. These results can inform those interested in employing CHWs in their CHW program development. Findings from this study can be used to guide development of tailored curriculum for continuing education units, specialized certifications, or other professional development resources for CHWs.
\end{abstract}

Keywords Community health workers $\cdot$ Workforce development $\cdot$ Promotores de salud $\cdot$ Employee engagement

\section{Introduction}

Promotores de Salud, or Community Health Workers (CHWs) are trusted members of the community who have a close understanding of the race, ethnicity, language, socioeconomic status and life experiences of the communities they serve [1]. CHWs are non-medical public health workers who connect communities to health care and social service providers. This connection enables them to reduce barriers

The authors would like to acknowledge Kaleigh Becker, MPH and Elena Martinez, MS for their help on the data analysis. We would like to thank all of the $\mathrm{CHW}$ employers who took the time to complete this survey.

Melissa Dunn

Melissa.Dunn@dshs.texas.gov

1 Health Promotion and Chronic Disease Prevention Section, Texas Department of State Health Services, 1100 W. 49th street T405.01, Austin, TX 78756, USA to accessing care for clients and allows for unrestricted access to communities. The term "CHW" is an umbrella term with a variety of job titles reflecting the diversity of the CHW profession: community health educator, health eligibility specialist, patient navigator, etc.

CHWs are employed in many types of settings, from health care facilities to community-based organizations, and provide various services to fit the needs of their community. CHWs fulfill a broad range of roles such as: access to health care services, outreach to encourage participation in health events, patient navigation, follow-up care and tracking referrals, culturally appropriate health and wellness education, informal counseling, social support, and advocacy for individual and community needs. CHWs network, build community connections, and provide cultural mediation between their communities and health and human service systems to reduce health care disparities. CHWs provide services to increase access to care through outreach and cultural linkages between communities and delivery systems. 
An ever-growing body of literature demonstrates that CHWs can effectively address chronic disease [2], improve medication-taking behavior [3], engage underserved communities [4], and reduce costs of primary and community care [5]. Numerous studies on CHW interventions, including on asthma control [6], diabetes management [7], and hospital-based supports [8], have all found positive return on investments.

In 2010, the U.S. Department of Labor's Bureau of Labor Statistics established a Standard Occupational Classification 21-1094 for CHWs. According to the May 2019 Occupational Employment Statistics Survey, there were 58,950 individuals employed as CHWs nationally. CHWs are employed in at least 49 states, Puerto Rico, and the District of Columbia. Texas is the fifth largest employer of CHWs (2900 in 2019) [9].

Texas has a long history of working to promote the CHW workforce in addressing health disparities. In 1999, Texas became the first state to adopt formal legislation to implement statewide training and credentialing standards for CHWs, regulating the CHW workforce. The Texas Department of State Health Services (DSHS) Promotor (a) or Community Health Worker Training and Certification Program (CHW program) implements a statewide certification process for CHWs and promotores, $\mathrm{CHW}$ instructors, and CHW training programs in Texas. The program approves qualified people as certified CHWs. Certification is based on completing a DSHS core competency certification course or through verified CHW experience of at least $1000 \mathrm{~h}$ in the last 3 years.

The CHW profession in Texas has grown significantly in the past 10 years. In December 2009 there were 625 certified CHWs in Texas; in December 2019, there were 4000 certified CHWs in Texas (this includes both people who are currently and not currently employed as CHWs) [10]. Since 2015 alone, the CHW profession in Texas has experienced an overall increase of $27 \%$. However, despite the growth in the CHW profession in Texas, there is still a lack of understanding of the CHW profession among employers.

There is ample literature about ways CHWs have been integrated into various programs and settings [1-8]. However, there are few articles that look at CHW employment context generally, from either a state or national level. Even more specifically, there is a gap in the literature about the employment contexts of Texas CHWs. Additionally, while there is some literature on the workforce development needs of CHWs in other U.S. states [11, 12], we found none that explored the workforce development needs that Texas CHW employers perceive for their CHW employees. As such, we conducted a survey of Texas CHW employers in order to fill this gap and better understand the CHW profession in Texas. The survey results include the perspective of $\mathrm{CHW}$ employers on CHW employment context, culturally-relevant services provided by CHWs, and workforce development needs.

\section{Methods}

A mixed-methods cross-sectional survey was developed for this study. The survey covered 11 domains including: CHWs' job responsibilities, compensation for CHWs, and workforce development. This paper presents the findings from six sections pertaining to CHW employment, training, and professional development, with a particular focus on the identified gaps in existing resources. The other five sections were about supervision, compensation, financing, challenges and benefits of employing CHWs, and evaluation of CHW interventions; data from these sections were not included in this paper as they were outside of the scope of this paper.

The survey originally contained 56 questions, including 11 open ended questions. The survey was pilot tested in 2018 by $65 \mathrm{CHW}$ employers from public health region (PHR) 2/3 North (north Texas including Dallas) and PHR 8 (central south Texas including San Antonio). The survey questions were edited for clarity, brevity, and structure following the pilot test. The final version of the survey consisted of 51 questions, including 8 open-ended questions.

\section{Procedure}

The survey was only available in English and was programmed using Qualtrics. A list of CHW employers was generated by accessing a database of employers through an online licensing system. An email with a link to the survey was sent to $841 \mathrm{CHW}$ employers across Texas. It is possible that some respondents were from the same organization. Not all CHW employers in Texas may have had their email address in the database.

The survey was open from February 10, 2020 to March 26, 2020. Survey reminder emails were sent on February 17, 2020 and March 9, 2020. During the survey collection period, 313 people started the survey. Of these, 65 respondents were not eligible to take the survey as they were not a CHW supervisor or manager, CHW employer, or CHW program coordinator or director. These respondents were exited from the survey and excluded from the analysis. Of the 248 respondents who met the survey eligibility requirements, 182 respondents completed the survey. Sixty-six eligible respondents were excluded from the analysis because they did not complete questions past the demographics section. The response rate of eligible respondents only $(n=776)$ was $23.5 \%$.

Of the 182 responses included in the analysis, some respondents did not provide answers to every question. For 
each question, the number of respondents ranged from 119 to 181 .

\section{Measures}

The findings in this report cover measures from six of the 11 sections in the survey.

Respondents were asked about various aspects of CHWs' employment at their organization, including how they are employed, their inclusion on interdisciplinary teams, and job duties. Respondents were also asked about the number of CHWs their organization employed and the number of people those CHWs served each month.

Respondents were asked a series of questions about the workforce development needs of their CHWs. In order to contextualize respondents' answers about workforce development needs, they were asked to "Describe how you assess the training needs of your CHWs." Respondents were also asked "To what extent does your organization currently have the capacity to implement the following workforce development activities?" and were presented with a 6-level Likert scale with responses from "Yes, to a great extent" to "No, not at all." Measures asked respondents if their
"DSHS [Texas Department of State Health Services]-certified CHWs employed by your organization complete any specialized training other than the 160 -h CHW credentialing course?" The final questions asked respondents about the specific training topics they needed and their workforce development needs.

\section{Data Analysis}

Descriptive statistics were calculated for all quantitative measures using Microsoft Excel and SPSS. Qualitative results from the open-ended questions were analyzed using grounded theory in N.Vivo and Microsoft Excel.

\section{Results}

\section{Employment Context}

As seen in Table 1, most respondents described their organization as hospitals/clinics ( $\mathrm{n}=28,15.5 \%)$, non-profit organizations ( $n=27,14.9 \%$ ), or federally qualified health centers (FQHC) $(n=24,13.3 \%)$. Over $75 \%(n=150)$ of respondents
Table 1 Type of organization and $\mathrm{CHW}$ educational requirements

\begin{tabular}{|c|c|}
\hline & $\mathrm{N}(\%)$ \\
\hline \multicolumn{2}{|c|}{ Which of the following best describes your organization? $(n=181)$} \\
\hline \multicolumn{2}{|l|}{ Organization type } \\
\hline Hospital/clinic & $28(15.5)$ \\
\hline Non-profit organization & $27(14.9)$ \\
\hline Federally qualified health care center (FQHC) & $24(13.3)$ \\
\hline Community-based organization & $18(9.9)$ \\
\hline Local health department & $16(8.8)$ \\
\hline Other & $14(7.7)$ \\
\hline College/university/school & $14(7.7)$ \\
\hline Health insurance plan & $13(7.2)$ \\
\hline Community health clinic & $12(6.6)$ \\
\hline State agency & $5(2.8)$ \\
\hline Area Health Education Center (AHEC) & $5(2.8)$ \\
\hline Health/social service organization & $5(2.8)$ \\
\hline \multicolumn{2}{|c|}{ How do CHWs participate in your organization? Select all that apply. $(n=182)$} \\
\hline \multicolumn{2}{|l|}{ Metric } \\
\hline Directly employ CHWs & $150(82.4)$ \\
\hline Other & $25(13.7)$ \\
\hline Contract CHWs (external) & $23(12.6)$ \\
\hline \multicolumn{2}{|c|}{ What are your organization's educational requirements for CHWs? Select all that apply. $(n=171)$} \\
\hline \multicolumn{2}{|l|}{ Education requirement } \\
\hline High school diploma or GED & $121(70.8)$ \\
\hline Work experience in lieu of education & $65(38.0)$ \\
\hline Some college & $31(18.1)$ \\
\hline College diploma & $29(17.0)$ \\
\hline Graduate degree & $15(8.8)$ \\
\hline
\end{tabular}


directly employed CHWs and $12.6 \%(n=23)$ of respondents contracted their CHWs externally. Of those who responded "Other," eight (4.4\%) indicated that their CHWs were current employees who offered CHW services in addition to their other job duties. Most of the respondents $(n=124$, $68.9 \%$ ) reported that CHWs were part of a multidisciplinary healthcare team, which was defined as a group of health care professionals from multiple disciplines working with a lead provider, typically a physician, nurse, or social worker.

Three-fourths of respondents $(n=136,75.1 \%)$ required their CHWs to be certified by the Texas Department of State Health Services (DSHS), while 9.9\% $(n=18)$ of respondents were not sure if their $\mathrm{CHW}$ were required to be certified. Most respondents $(n=121,70.8 \%)$ required their CHWs to have at least a high school diploma or GED (Table 1). Fewer respondents $(n=65,38.0 \%)$ said that their CHWs could also have work experience in lieu of their standard education requirements.

The mean number of CHWs employed by respondents' organization was $15 \mathrm{CHWs}$ and the mode was 2 CHWs (range 1-550). The mean number of people reached by organizations' CHWs each month was 531 and the mode was 150 people (range $0-8000$ ).

Respondents indicated that their CHWs delivered a variety of services (Table 2). The most commonly cited services were health education/promotion $(n=126,85.7 \%)$, information and referral $(\mathrm{n}=121,82.3 \%)$, and CHW services in community-based settings $(n=114,77.6 \%)$. Of the four respondents who selected "Other," responses included "provision of needs" and "health care benefits". The main health issues addressed by CHWs were diabetes $(n=106$, $67.9 \%)$, hypertension $(\mathrm{n}=90,57.7 \%)$, and mental/behavioral health $(n=86,55.1 \%)$. The main social barriers addressed by CHWs were transportation $(\mathrm{n}=139,89.7 \%)$, lack of health insurance $(\mathrm{n}=138,89.0 \%)$, and connecting to health resources $(n=136,87.7 \%)$.

\section{Workforce Development Needs}

Respondents used several strategies to determine the workforce development needs of their CHWs. Among answers provided by 73 respondents, $38.4 \%$ said that they looked at CHW scope and project needs $(\mathrm{n}=28)$ to determine workforce development needs. Others met with CHWs and discussed their training needs $(n=19,26.0 \%)$ or determined needs based on observing the CHW within the community $(n=10,13.7 \%)$. Some respondents left the workforce development needs to their CHWs, either selecting continuing education classes based on the CHWs credentialing requirements $(n=13,17.8 \%)$ or the class qualities, such as access, cost, or topic $(\mathrm{n}=5,6.8 \%)$.

Respondents identified many workforce development needs for their CHWs (Table 3). Over half of respondents indicated that the key workforce development needs included "Continued training to update CHWs on new skills, to reinforce initial training, and to ensure they are practicing skills learned" $(n=67,56.3 \%)$ and "Development of long-term financial mechanisms to fully support CHW strategies" $(n=65,54.6)$. Over half of respondents $(n=62,52.1 \%)$ also indicated that their CHWs would benefit from specialized continuing education. Some respondents also included "CHW credentialing through completion of the 160-h CHW course required by DSHS" $(n=24,20.2 \%)$ as a workforce development need.

The capacity to implement different workforce development activities differed between activities and employers (Fig. 1). The activities that respondents had the greatest capacity to implement included "Apply standards for training and supervision of CHWs" ( $\mathrm{n}=66,55.5 \%)$, "Support continued development of skills of CHWs" $(n=62,53.0 \%)$, and "Incorporate a full range of CHW competencies/skills in the CHW positions" $(\mathrm{n}=63,54.3 \%)$. The activities that respondents had the least capacity to implement were "Compensate for attendance/travel to state and national $\mathrm{CHW}$ conferences and CHW Association membership" $(\mathrm{n}=35$, $31.3 \%$ ), "Compensate for certified continuing education for CHWs provided by DSHS" ( $\mathrm{n}=44,38.6 \%)$, and "Develop career pathways for CHWs" $(n=44,39.3 \%)$.

Expanding on the need for "continued training" and specialized continuing education, respondents identified the general health training topics that would be beneficial for their organizations' CHWs (Table 3). Nearly half of respondents said that their CHWs would benefit from additional training in chronic disease self-management $(n=61,49.6 \%)$. The other frequently selected topics included health promotion $(\mathrm{n}=58,47.2 \%)$, case coordination/patient navigation $(\mathrm{n}=55,44.7 \%)$, and mental health $(\mathrm{n}=52,42.3 \%)$. Many CHWs were already engaged in additional specialized trainings or continuing education programs.

To become a DSHS-certified CHW, the CHW must take a required 160-h credentialing course; however, CHWs may take specialized trainings in addition to this. Of 121 respondents, 54.5\% ( $n=66)$ said their DSHS-certified CHWs had completed specialized training other than the required 160-h credentialing course. Among those respondents, 51 people provided qualitative descriptions of the specialized training. From most to least commonly stated, the specialized trainings included:

1. Physical and mental health training (i.e. chronic disease, motivational interviewing, reproductive health, harm reduction) $(\mathrm{n}=25)$;

2. CHW-specific training and health national standards $(n=14)$;

3. Reproductive health $(\mathrm{n}=5)$; and

4. Child and infant nutrition and safety $(n=4)$. 
Table 2 Services delivered and populations served by $\mathrm{CHWs}$

\section{$\mathrm{N}(\%)$}

What types of services do your organization's CHWs deliver? Select all that apply. $(n=147)$

Service

Health education/promotion

$126(85.7)$

Information and referral

$121(82.3)$

CHW services in community-based settings

$114(77.6)$

System navigation/access to services

$110(74.8)$

Individual and community advocacy

$94(63.9)$

Informal counseling and social support

$74(50.3)$

CHW Services in clinical settings

69 (46.9)

Direct services

$62(42.2)$

Cultural liaison/mediation

$36(24.5)$

Other

$4(2.7)$

What are the main health issues of the populations addressed by your organization's CHWs?

Select all that apply. $(n=156)$

Diagnosis

Diabetes

$106(67.9)$

Hypertension

$90(57.7)$

Mental/behavioral health

$86(55.1)$

Obesity

76 (48.7)

Heart disease

$73(46.8)$

Other health issues/chronic disease

$71(45.5)$

Maternal and child health

$62(39.7)$

Domestic and sexual violence

$52(33.3)$

Sexual health

$50(32.1)$

Substance abuse

$49(31.4)$

Cancer

$48(30.8)$

Asthma

$47(30.1)$

Social isolation

$41(26.3)$

HIV/AIDS

37 (23.7)

34 (21.8)

Oral health

33 (21.2)

Falls prevention

29 (18.6)

Substance abuse (opioids)

$16(10.3)$

Other

$14(9.0)$

What are the social barriers of populations served by your organization's CHWs? Select all that apply. $(\mathrm{n}=155)$

Barrier

Transportation

$139(89.7 \%)$

Lack of health insurance

$138(89.0 \%)$

Connecting to health resources

$136(87.7 \%)$

Limited English skills

$124(80.0 \%)$

Food Insecurity

$117(75.5 \%)$

Housing

$116(74.8 \%)$

Homelessness

$88(56.8 \%)$

Other
Despite the identified unfilled workforce development needs, $65 \%$ of respondents $(n=78)$ said they would be willing to expand $\mathrm{CHW}$ employment at their organization in the future.

\section{Discussion}

The current study explored the employment context and workforce development needs of community health workers 
Table 3 CHW workforce development needs

What are your organization's main CHW workforce development needs? Select all that apply. $(\mathrm{n}=119)$

Workforce Development Need

Continued training to update CHWs on new skills, to reinforce initial training, and to ensure they are practicing skills learned

Development of long-term financial mechanisms to fully support CHW strategies

$67(56.3)$

Specialized continuing education for CHWs

65 (54.6)

Training on CHW role, and benefits to clients, the care team and organization

$62(52.1)$

Training to equip supervisors with skills to effectively oversee and support CHW services

Development of organizational resources

Networking and membership in one of 12 local and regional CHW associations and coalitions across the state of Texas

Building workforce development collaborative with DSHS-certified training centers for CHWs

CHW credentialing through completion of the 160-h CHW course required by DSHS

Other

Which of the following general health training topics would be most beneficial for your organization's CHWs? Select up to 3 responses. $(\mathrm{n}=123)$

Training Topic

Chronic disease self-management

Health promotion

Case coordination/patient navigation

Mental health

Prevention/screening cholesterol, diabetes, high blood pressure

Outreach

Women's health

Care coordination

Program management and policy administration

Insurance

Home care training [home visiting]

Infectious disease

Children/adolescent health

Oral health

Other

Not sure/unknown

throughout Texas. The first aim of the study was to understand the employment context of CHWs in Texas. We found that most CHW employers directly employed their CHWs, and CHWs were typically part of a multidisciplinary healthcare team. Most respondents required their CHWs be DSHScertified and have at least a high school diploma or GED.

We also found that there was tremendous variation in the number of CHWs employed by each organization and the number of people served by those CHWs each month. In line with previous literature, the most common services that $\mathrm{CHW}$ provided were health education/ promotion, information referral, and CHW services in community-based settings $[11,13,14]$. More than in previous literature, our study found that the main health issues CHWs addressed were diabetes, hypertension, and mental/behavioral health [14]. CHWs also addressed various social barriers, such as transportation, lack of health insurance, and connecting to health resources.

The second aim of this study was to ascertain the workforce development needs of CHW employers in Texas. We found that $\mathrm{CHW}$ employers needed specific, specialized training for their CHWs, such as education about physical health or reproductive health. Additionally, CHW employers reported a need for physical and mental health self-management resources. Specifically, the findings suggest that CHWs could most benefit from education on emerging health topics to supplement the required certification training (Table 3 ). These findings are consistent with previous literature on the topic: a statewide survey of CHW employers $(n=240)$ conducted in the American Midwest indicated a need for training opportunities for CHWs, which included "ongoing training" (79\%) and "mandatory training" (78\%) [12]. This 
Fig. 1 Extent to which organizations currently have the capacity to implement workforce development activities

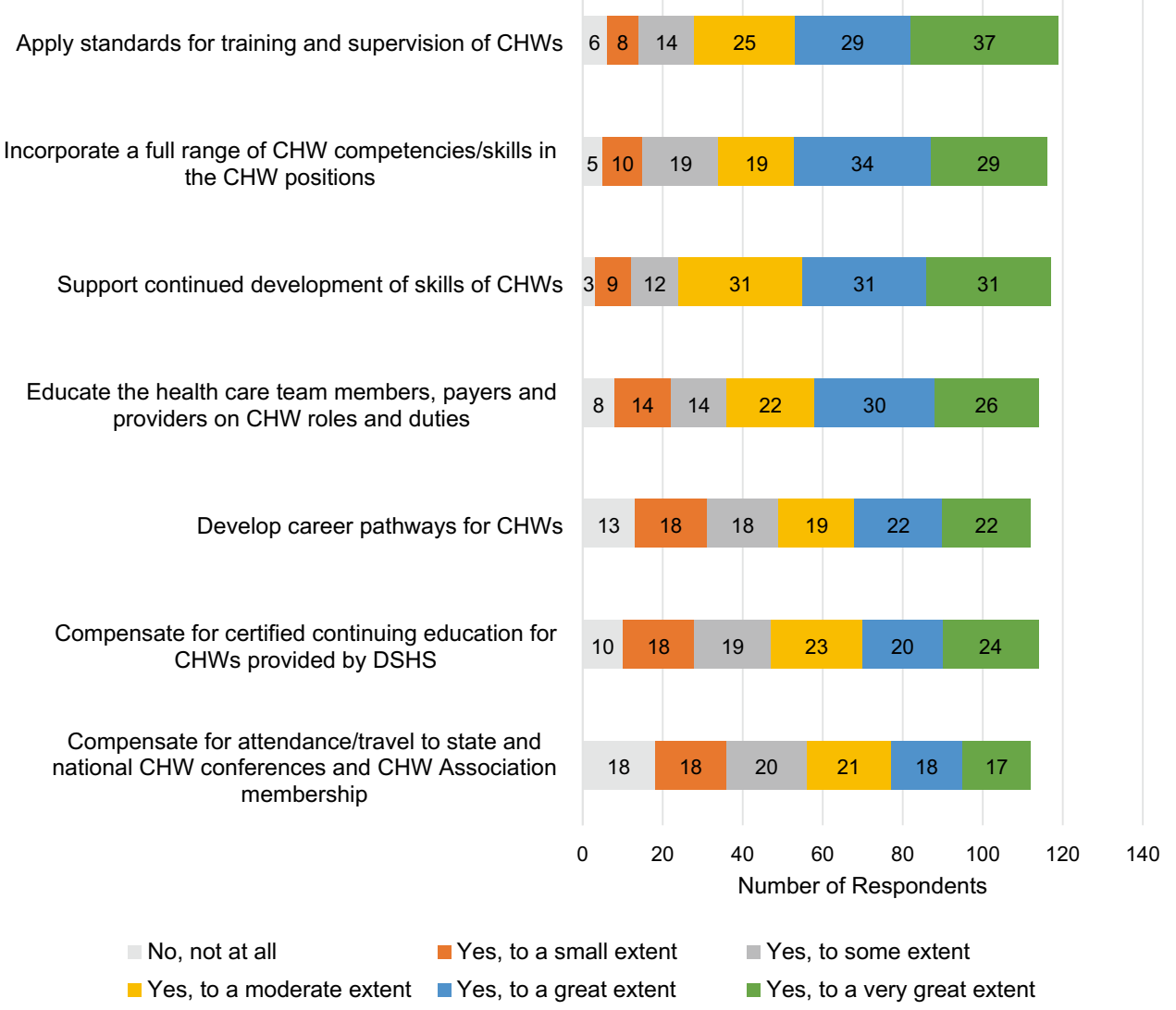

implies that the services rendered by CHWs require skills and knowledge for which CHWs need specialized training.

The findings from this survey also document important details regarding the extent to which employers have the capacity to implement workforce development activities. For each workforce development activity, more than $25 \%$ of respondents reported having capacity to a "great extent" or a "very great extent" to implement (Fig. 1) different activities. This data is relevant to understanding the potential for organizations to adopt and implement workforce development resources. Each category had at least $25 \%$ of respondents reporting some level of ability to implement activities, implying that if workforce resources were available, organizations would be able to use them.

Importantly, the survey findings described the workforce development activities that respondents had the least capacity to implement. These included: "Compensate for attendance/travel to state and national CHW conferences and CHW Association membership" $(\mathrm{n}=35)$ and "Develop career pathways for CHWs" $(n=43)$. The latter point is consistent with findings from a survey of CHW employers in all 50 states conducted in 2007, which found "the occupation of CHW has not been viewed as a career, because CHWs have positions that are often short-term, low paid, and lack recognition by other professionals" [14].
Over the past decade, the CHW profession has evolved significantly. Moreover, 19 states have state-operated or privately-operated certification program for CHWs. Another 18 states have a framework for CHW credentialing under consideration or under development [15]. In 2019, the National Association of CHWs (NACHW) was launched with a mission to "unify the voices of CHWs and strengthen the profession's capacity to promote healthy communities". As of 2020, Texas has its own state Texas Association of Promotores and CHWs (TAPCHW). But despite these advancements, the findings in this study show clear challenges CHW employers still face in effectively and efficiently employing and developing CHWs.

\section{Public Health Implications}

These findings have important implications for CHWs, their employers, and the populations they serve. The surveyed CHW employers reported a strong need for resources to train CHWs on specific topic areas, yet also identified having limited capacity to provide "career pathways" for their CHWs. Together, these findings imply that employers recognize the need for CHWs to receive specific, supplemental training, while also understanding their limitation to provide that training independently. Furthermore, these findings suggest 
that, without increased educational and workforce development opportunities, the CHW profession will continue to experience inhibited career prospects.

One approach to addressing these challenges is to retrofit the general CHW certification with a specialized or specialty CHW certification. This would support employers with $\mathrm{CHW}$ job design, selection and recruitment of $\mathrm{CHW}$ with specialized skills and knowledge, CHW career advancement and integration of CHWs in healthcare teams and the CHW practice. Another approach would be to create cross-sector partnerships with employers geared towards developing cultural competency trainings for CHW supervisors and trainings to equip supervisors with skills to effectively oversee and support CHW services and increase awareness of the CHW roles among employers.

Findings from this study can be used by state health departments or universities to develop tailored curriculum for continuing education units (CEUs), specialized certifications, or other professional development resources for CHWs. If specialized certifications are not possible, these stakeholders could consider incorporating some of the identified topics into the required certification curriculum to increase the foundational competency of CHWs. Furthermore, this study identifies a potential opportunity for CHWcertifying bodies and CHW employers to address a resource gap through collaboration. For example, CHW-certifying bodies could serve an identified need by developing and distributing professional development resources through strategic partnerships with CHW employers.

\section{Strengths and Limitations}

Study findings should be interpreted in the context of at least two limitations. The first limitation of this study is the sampling. The distribution list of CHW employers was generated by accessing a database of employers through an online licensing system. It is possible that this list did not contain email addresses for every CHW employer in Texas, and it is unknown if those not included are materially different from those who were included. Additionally, in order to protect the anonymity of the respondents, we did not ask them to identify their organization. As such, it is also possible that some respondents were from the same organization.

The second limitation is the response rate. The survey was fielded from February 14, 2020-March 16, 2020, right at the beginning of the COVID-19 pandemic in the United States. It is possible that the response rate was affected by CHW employers' need to focus on the evolving pandemic.

Despite these limitations, the study has notable strengths. The survey tool was pilot tested with 65 CHW employers and significantly revised based on their feedback. These revisions helped us ensure our survey tool was as streamlined and clear, yet comprehensive as possible, providing for rich data. Additionally, this study adds to the literature on Texas CHW employers' identified workforce development needs, which does not currently exist. This study also contributes to existing literature about the resources CHWs need to improve the professional competencies of the workforce.

\section{Suggestions for Future Research}

This study relied on the responses of CHW employers, but not CHWs or patients. Further research should consider including CHWs and patients in surveys or qualitative inquiries to understand their perspectives and nuance on resources or trainings needed. Additionally, the survey tool was developed prior to the COVID-19 pandemic. Future research should examine the impact of COVID-19 on CHW workforce development needs, such as topic areas for training and employers' capacity to support workforce development activities.

\section{Conclusion}

We conducted a survey of $182 \mathrm{CHW}$ employers in Texas to understand the employment context and workforce development needs of community health workers throughout Texas. We found that most CHW employers directly employed their CHWs, and CHWs were typically part of a multidisciplinary healthcare team. Most respondents required their $\mathrm{CHWs}$ be DSHS-certified and have at least a high school diploma or GED.

There was tremendous variation in the number of CHWs employed by each organization and the number of people served by those CHWs each month. The most common services that CHWs provide included health education/ promotion, information referral, and CHW services in communitybased settings. The main health issues that CHWs addressed were diabetes, hypertension, and mental/behavioral health. CHWs also addressed various social barriers, such as transportation, lack of health insurance, and connecting to health resources.

To determine workforce development needs, CHW employers considered their CHWs' scope and project needs. Current workforce development needs included continued training on various topics, including chronic disease selfmanagement and health promotion. CHW employers also report needing a long-term financial mechanism to support CHWs. CHW employers differed in their capacity to implement workforce development activities. Results reinforce previous findings on the need for specialized, continued training for CHWs and the development of pathways, resources, and opportunities that could establish it as a profession. 
Acknowledgements The authors would like to acknowledge Kaleigh Becker, MPH and Elena Martinez, MS for their help on the data analysis. We would like to thank all of the CHW employers who took the time to complete this survey.

Author Contributions MD: Design of the research study, data collection, analyzed data and wrote the manuscript. EJ: Analyzed quantitative data, and helped with manuscript writing, BS: Design of the research study, data collection and provided substantial revisions to the introduction and results sections of the manuscript. MC: Design of the research study and contributed substantial revisions to the content of all sections of the manuscript. NB: Design of the research study and contributed substantial revisions to the content of all sections of the manuscript.

\section{Funding Not applicable.}

Data Availability Deidentified data is available by request with a data use agreement (DUA) and subject to legal approval. Data tables for aggregate data is available upon request. The survey tool is available upon request.

Code Availability Quantitative software programs used were Microsoft Excel and SPSS.

\section{Declarations}

Conflict of interest The authors declare that there are no financial, consultant or institutional conflicts of interest.

Informed Consent Participation in the survey is voluntary and anonymous. The survey will only take about $20 \mathrm{~min}$ to complete. You can skip any question you do not wish to answer.

\section{References}

1. Community Health Workers. American Public Health Association (APHA). Retrieved January 12, 2021, from https://www.apha.org/ apha-communities/member-sections/community-health-workers/

2. Guide to Community Preventive Services. (March 2015). Cardiovascular disease: Interventions engaging community health workers. Retrieved December 9, 2020, from https://www.thecommuni tyguide.org/findings/cardiovascular-disease-prevention-and-contr ol-interventions-engaging-community-health. Page last updated: September 30, 2020.

3. Brownstein, J. N., Chowdhury, F. M., Norris, S. L., Horsley, T., Jack, L., Zhang, X., \& Satterfield, D. (2007). Effectiveness of community health workers in the care of people with hypertension. American Journal of Preventive Medicine, 32(5), 435-447. https://doi.org/10.1016/j.amepre.2007.01.011.

4. Palmas, W., Teresi, J. A., Findley, S., Mejia, M., Batista, M., Kong, J., Silver, S., Luchsinger, J. A., \& Carrasquillo, O. (2012). Protocol for the Northern Manhattan Diabetes Community Outreach Project. A randomised trial of a community health worker intervention to improve diabetes care in Hispanic adults. British Medical Journal Open, 2(2), e001051. https://doi.org/10.1136/ bmjopen-2012-001051.
5. Johnson, D., Saavedra, P., Sun, E., Stageman, A., Grovet, D., Alfero, C., Maynes, C., Skipper, B., Powell, W., \& Kaufman, A. (2012). Community health workers and Medicaid managed care in New Mexico. Journal of Community Health, 37(3), 563-571. https://doi.org/10.1007/s10900-011-9484-1.

6. Sustainable Financing Models for Community Health Worker Services in Maine. (2016). Prepared by Center for Health Law and Economics, University of Massachusetts Medical School. Retrieved from https://commed.umassmed.edu/sites/default/files/ publications/Sustainable\%20Financing $\% 20 \mathrm{ME} \% 20 \mathrm{CHWs} \% 20-\%$ 20UMass\%20Report\%20Nov\%202016\%20Final.pdf

7. London, K., Love, K., \& Tikkanen, R. (2017). Sustainable Financing Models for Community Health Worker Services in Connecticut: Translating Science into Practice. Prepared by Center for Health Law and Economics, University of Massachusetts Medical School. Retrieved from https://www.cthealth.org/wp-content/ uploads/2017/06/CHF-CHW-Report-June-2017.pdf

8. Kangovi, S., Mitra, N., Grande, D., Long, J. A., \& Asch, D. A. (2020). Evidence-based community health worker program addresses unmet social needs and generates positive return on investment. Health Affairs (Millwood)., 39(2), 207-213.

9. Occupational Employment and Wages, May 2017: 21-1094 Community Health Workers. (2018, March 30). Bureau of Labor Statistics. Retrieved January 4, 2021, from https://www.bls.gov/oes/ 2017/may/oes211094.htm\#nat

10. Promotora or Community Health Worker Training and Certification Program. (2019). 2019 Annual Report: Promotora or Community Health Worker Training and Certification Program. Texas Department of State Health Services, Health Promotion and Chronic Disease Prevention Section. Retrieved from https://www. dshs.state.tx.us/chw/docs/2019CHWAnnualReport.pdf

11. Sugarman, M., Ezouah, P., Haywood, C., et al. (2021). Promoting community health worker leadership in policy development: Results from a Louisiana Workforce Study. Journal of Community Health, 46, 64-74. https://doi.org/10.1007/s10900-020-00843-7.

12. Chaidez, V., Palmer-Wackerly, A. L., \& Trout, K. E. (2018). Community health worker employer survey: Perspectives on $\mathrm{CHW}$ workforce development in the Midwest. Journal of Community Health, 43(6), 1145-1154. https://doi.org/10.1007/ s10900-018-0533-x.

13. Rosenthal, E. L., Wiggins, N., Ingram, M., Mayfield-Johnson, S., \& De Zapien, J. G. (2011). Community health workers then and now: An overview of national studies aimed at defining the field. Journal of Ambulatory Care Management., 34(3), 247-259. https://doi.org/10.1097/JAC.0b013e31821c64d7.

14. U.S. Dept of Health and Human Services. (March 2007). Community health worker national workforce study. Retrieved from https://bhw.hrsa.gov/sites/default/files/bureau-health-workforce/ data-research/community-health-workforce.pdf

15. Centers for Disease Control and Prevention. (2016). State Law Fact Sheet: A summary of state community health worker laws (CDC Publication No. CS2770666). U.S. Department of Health and Human Services, Centers for Disease Control and Prevention. Retrieved from https://www.cdc.gov/dhdsp/pubs/docs/SLFSSummary-State-CHW-Laws.pdf

Publisher's Note Springer Nature remains neutral with regard to jurisdictional claims in published maps and institutional affiliations. 